\title{
An unusual clinical presentation of lupus vulgaris mimicking a vascular malformation in a Spanish old
} Woman

\author{
Ma Reyes García de la Fuente*, Mercedes Pérez, Petiti GH and González A \\ Department of Dermatology, Hospital Moisès Broggi-General. Av. Josep Molins, 29-41, 08906, L’Hospitalet de Llobregat, Barcelona, Spain
}

\begin{abstract}
Tuberculosi is an infectious disease produced by Mycobacterium tuberculosis that mainly affected the lungs. Cutaneous tuberculosis accounts for $1,5 \%$ of extrapulmonary tuberculosis. Lupus vulgaris is the most common form of cutaneous tuberculosis, mostly involving the head and neck region. It has the potential of autoinoculation and can spread to other areas of the body. Ear are typically affected, an enlarged earlobe with bluish-red or violaceous indurated plaques and nodules, can be a sign of lupus vulgaris. We are reporting a case of a 86 years old female with a big violaceous plaque in neck, extending to right ear, that has been growing for more than thirty-five years.
\end{abstract}

Another violaceous plaque with a similar evolution was discovered on her left leg during the anamnesis. A misdiagnosis of vascular malformation was given by another practionner, more than ten years ago.

Physical examination was normal and systemic symptoms were absent, but skin biopsy and cultural analysis revealed a diagnosis of lupus vulgaris.

We report a case of lupus vulgaris, an entity of a very low frequency in our country, to emphasize the importance of having it in mind to make the differential diagnosis.

\section{Introduction}

Tuberculosis (TB) is a disease caused by Mycobacterium tuberculosis that affects various organs and remains one of the world's deadliest diseases. TB has surpassed AIDS as the leading cause of death due to infectious disease ${ }^{1}$. The main infected organ is lungs. The tuberculosis decreased in the 1980s because of the BCG vaccine and improvements in public health care and anti-TB chemotherapy. However, since 1985, the tuberculosis cases have gradually increased due to the increase of the global population and increasing amount of HIV positive and multiple drug-resistant TB patients. According to a World Health Organization (WHO) report, TB accounted for 10,4 million new cases and 1,8 million deaths worldwide in 2015 [1]. The TB extrapulmonary infection reaches up to $14 \%$, among which the most common sites include the lymph nodes, peritoneum, ileocecal, hepatosplenic, genitourinary, central nervous system, and musculoskeletal regions [2]. Diagnosis of TB in these organs is often overlooked because of its atypical clinical manifestations. However, extrapulmonary tuberculosis (EPTB) still contributes to a substantial TB incidence, and under- diagnosis of EPTB is also likely to cause life-long sequelae and fatal complications $[3,4]$. Head and neck tuberculosis (HNTB) accounts for $10 \%$ of TB patients [5]. HNTB can affect most organs in the head and neck region, such as the lymph nodes, larynx, middle ear, oral cavity and pharynx [6]. In general, entrance of $M$. tuberculosis into these regions is covered with epithelium mucosa; therefore, immunosuppression or a break in this natural barrier caused by trauma, inflammation, poor oral hygiene.

Cutaneous TB is uncommon (1,5\% of all cases of tuberculosis) [7]. It presents a wide variety of clinical forms depending on how bacteria reach the skin and on the immune status of the patient. In
Spain, as most of industrialized countries, cutaneous tuberculosis is very rare and the majority of cases are reported in immigrants or in elderly people who had contracted tuberculosis long time ago whereas younger people are mostly affected in developing countries [8].

\section{Case report}

A 86-year-old Spanish woman, with a clinical history of hypertension and Alzheimer disease and non- clinical history of immunosuppression was referred to our service due to an asymptomatic cutaneous lesion that appeared about thirty-five years ago and had progressively enlarged over time.

Ten years ago the patient had contacted with another practionner for the same lesion and a clinical diagnosis of vascular malformation was given. The cutaneous lesion consisted in a non-tender, violaceous plaque in her neck extending to right ear (Figure 1).

A skin biopsy was made from the right cheek side and the histopathological examination revealed an abundant tuberculoid granulomas containing Langhans-type giant cells in dermis (Figure $2 \mathrm{~A}$ and $2 \mathrm{~B}$ ). The staining for acid-fast bacilli was positive for a Mycobacterium spp. (Figure 2C). Cultural analysis of the skin biopsy

${ }^{\star}$ Correspondence to: $M^{\text {a }}$ Reyes García de la Fuente, ORCID: 0000-0002-70703110, Av. Josep Molins, 29-41, 08906. L'Hospitalet de Llobregat, Barcelona, Spain, Tel: +34 9355312 00; E-mail:mrgarcia_dlf@hotmail.com

Key words: Cutaneous infection, cutaneous tuberculosis, extrapulmonary tuberculosis, lupus vulgaris, Mycobacterium tuberculosis.

Received: April 13, 2020; Accepted: April 21, 2020; Published: April 24, 2020 
was positive for Mycobacterium tuberculosis. During the physical exploration another violaceous plaque in her left leg was revealed with similar features to the facial lesion and similar results were found out in the biopsy practionned (Figure 3 ). The patient was asymptomatic, no fever, no weight lost, and any other systemic manifestation was detected, no past history of pulmonary TB. There was no history of trauma. She was not vaccinated with BCG. The patient had always lived in Spain and had never traveled abroad. No other familiars were affected. A chest X-ray did not show any sign of TB infection. The Quantiferon TB GOLD test was positive $(0,70 \mathrm{UI} / \mathrm{mL}$ IFNy to Mycobacterium tuberculosis). Baciloscopia was negative. These results were evidence of cutaneous TB disease, specifically the lupus vulgar form.

Antibiotical therapy was offered but the patient's family rejected that due to the side effects. The patient continues being asymptomatic even though she had not been treated.

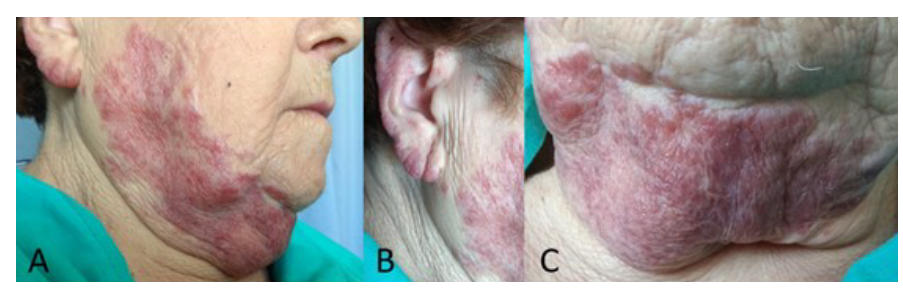

Figure 1. Erythematous plaque in right cheek and neck extending up to the right ear

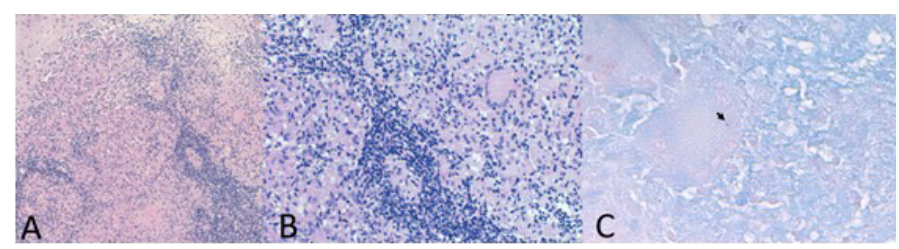

Figure 2. (A, B) The histological study revealed a Langhans giant cells surrounded by a dense inflammatory and chronic infiltrates with epithelioid cells and central necrosis in the dermis (A: H\&E, X100; B: H\&E, X20). (C) Ziehl-Neelsen staining showing a bacilli (arrow) compatible with Mycobacterium spp. (X100)

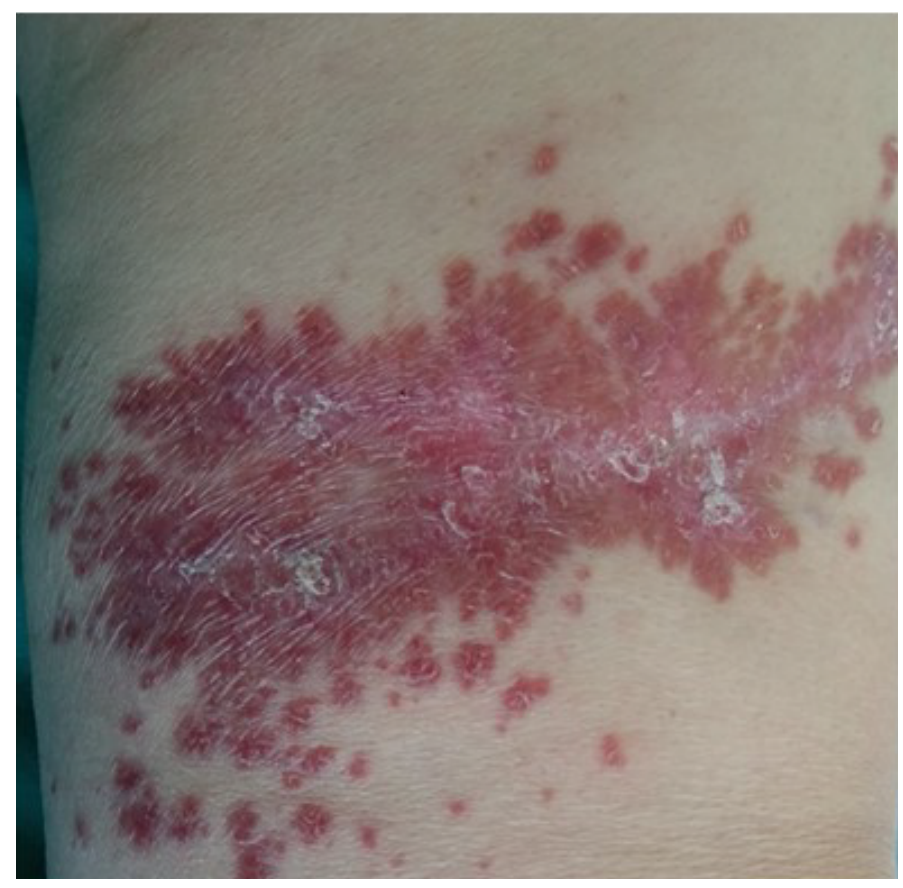

Figure 3. Erythematous plaque in left leg with a high number of spread lesions

\section{Discussion}

Tuberculosis is one of the commonest chronic granulomatous infections caused mainly by Mycobacterium tuberculosis but other bacilli in a very low percentage could be responsible as Mycobacterium bovis, Mycobacterium africanum or Mycobacterium microti. The diagnosis is difficult, due to the low frequency of the disease and the different ways of presentation. The frequency of extrapulmonary TB is low and the cutaneous infection is even lower as we commented in the introduction and the most implicated pathogens are Mycobacterium tuberculosis but Mycobacterium bovis and BCG vaccine (an attenuated strain of Mycobacterium bovis) could be responsible.

Cutaneous TB can be acquired from hematogenous or lymphatic dissemination of a pulmonary focus or by direct inoculation. The pivotal factor for the clinical presentations prior to contact with bacilli is the host natural immune response. Cutaneous TB could be classified according to different criteria as the route of infection or based on a load of pathogens.

According to the route of infection cutaneous TB is classified in exogenous source or endogenous source and tuberculids. The exogenous cutaneous $\mathrm{TB}$ is an infection that occurs with direct inoculation of bacilli into the skin of predisposed individuals. The main entities are tuberculosis chancre and tuberculosis verrucosa cutis. The endogenous cutaneous TB is secondary to a preexisting primary focus and may result from contiguous (orificial tuberculosis, scrofuloderma), hematogenous (acute miliary tuberculosis, tuberculous gumma, and lupus vulgaris), or lymphatic dissemination (lupus vulgaris). Scrofuloderma is the most common form of cutaneous TB in India, often affects the supraclavicular area, the axilla, and both sides of the neck [9]. Scrofuloderma is a type of secondary TB which occurs by contiguous spread from tuberculosis nodes, bones, or joints. These cases presented with discharging sinuses overlying caseating tuberculosis lymph nodes. Tuberculids include lichen scrofulosorum, papular or papulonecrotic tuberculide, erythema nodosum and erythema induratum of Bazin.

Lupus vulgaris (LV) is a chronic progressive form of tuberculosis, occurring in patients with moderate to high degree of immunity, originating from an underlying tuberculosis focus or through exogenous inoculation or following BCG vaccination so can occur due to both, exogenous and endogenous, source of infection [10] and it is the most frequent form of presentation of cutaneous TB in Europe [11]. The typical forms of LV are plaque form, ulcerative, mutilating, vegetative, tumour-like and the papular/ nodular form affecting the facial area and neck as the case reported. The face is the most commonly affected site in western countries with a frequent affliction of the nose, ear and cheeks. However, in India lower extremities, especially buttocks, are the most frequently affected areas and the relatively low frequency of facial lesions when compared to western world can be attributed to higher temperatures prevailing in most parts of India [12]. In the case reported, there were two focus of lupus vulgaris, in head and in left leg, so we think that the focus was endogenous in spite of the general study of health was normal with no signs of chest infection in the chest X-ray but with a positive Quantiferon test.

According to the load of pathogens cutaneous TB could be classified in multibacillary forms such as tuberculous chancre, scrofuloderma, orificial tuberculosis, acute miliary tuberculosis and tuberculous gumma and in a paucibacillary forms including TB verrucosa cutis, tuberculoid, and lupus vulgaris [10]. In fact, in the case reported, only one bacilli was detected in the biopsy (Figure 2C) as a paucibacillary form of cutaneous TB. 
The cutaneous TB treatment is often the same that pulmonary TB with a long and combined regime of antibioticals including rifampicin, isoniazid, ethambutol and pyrazinamide during two months and four months continuation including rifampicin and isoniazid [13]. In some cases a surgical drain is required in cutaneous TB forms that involve cold abscess.

Nowadays, the presence of two plaques of lupus vulgaris in a different anatomical area is very infrequent in Spanish population.

In Spain, an industrialized country, lupus vulgaris is very rare, and the few annual cases detected, were especially observed in elderly patients. So that, in order not to delay the diagnosis, to study the disease contagiousness and to avoid new cases, this entity should be especially considered in elderly patients.

Actually, the misdiagnosis of vascular malformation given to the patient ten years ago could have been avoided if it would have been of prime importance to keep cutaneous tuberculosis as a differential diagnosis in chronic skin condition with varied presentation.

\section{Conflicts of interest}

The authors have nothing to disclose

\section{References}

1. WHO (2019) Global tuberculosis report 2019. World Health Organization. WHO, Geneva, Switzerland.

2. Gunawan H, Achdiat P, Hindritiani R, Essary ED, Ningtias LD, et al. (2018) Various Cutaneous Tuberculosis with Rare Clinical Manifestations: A Case Series. Int $J$ Mycobacteriol 7: 288-291.
3. Sandgren A, Hollo V, van der Werf MJ (2013) Extrapulmonary tuberculosis in the European union and European economic area, 2002 to 2011. Euro Surveill 18: 20431. [Crossref]

4. Kulchavenya E (2014) Extrapulmonary tuberculosis: are statistical reports accurate? Ther Adv Infect Dis 2: 61-70. [Crossref]

5. Srivanitchapoom C, Sittitrai P (2016) Nasopharyngeal tuberculosis: epidemiology, mechanism of infection, clinical manifestations, and management. Int J Otolaryngol 2016: 4817429. [Crossref]

6. Das S, Das D, Bhuyan UT, Saikia N (2016) Head and neck tuberculosis: scenario in a tertiary care hospital of North Eastern India. J Clin Diagn Res 10: Mc04-Mc07. [Crossref]

7. Khadka P, Koirala S, Thapaliya J (2018) Cutaneous Tuberculosis: Clinicopathologic Arrays and Diagnostic Challenges. Dermatology Research and Practice. Dermatol Res Pract 2018: 7201973. [Crossref]

8. Pföhler C, Klotz M, Wehler T, Vogt T, Müller CSL (2017) A Slowly Growing Orange Patch on the Cheek: Diagnosis of Lupus Vulgaris 20 Years After Onset of First Skin Changes. Dermatol Ther (Heidelb) 7: 181-185. [Crossref]

9. Chopra D, Chopra V, Sharma A, Chopra S, Aggarwal S, et al. (2017) Unusual Sites of Cutaneous Tuberculosis: A Report of Two Cases. Case Rep Dermatol Med 2017 : 7285169. [Crossref]

10. Khadka P, Koirala S, Thapaliya J (2018) Cutaneous Tuberculosis: Clinicopathologic Arrays and Diagnostic Challenges. Dermatol Res Pract 2018: 7201973. [Crossref]

11. Striegel AK, Nazzal R, Fabri M, Rietschel E, Dötsch J, et al. (2014) Two cases of lupus vulgaris in childhood and review of the clinical challenges. Klin Padiatr 226: 40-3.

12. Padmavathy L, Rao LL, Ethirajan N, Krishnaswami B (2007) Ulcerative lupus vulgaris of face: an uncommon presentation in India. Indian J Tuberc 54: 52-54. [Crossref]

13. Wang H, Wu Q, Lin L, Cui P (2011) Cutaneous tuberculosis: a diagnostic and therapeutic study of 20 cases. $J$ Dermatolog Treat 22: 310-314. [Crossref]

Copyright: (C2020 García de la Fuente MR. This is an open-access article distributed under the terms of the Creative Commons Attribution License, which permits unrestricted use, distribution, and reproduction in any medium, provided the original author and source are credited. 